Improved capture gamma-ray libraries for nuclear applications

A. M. Hurst, B. W. Sleaford, R. B. Firestone, N. C. Summers, Z. Revay, L. Szentmiklosi, S. Basunia, T. Belgya, J. E. Escher, M. Krticka

October 11, 2012 
This document was prepared as an account of work sponsored by an agency of the United States government. Neither the United States government nor Lawrence Livermore National Security, LLC, nor any of their employees makes any warranty, expressed or implied, or assumes any legal liability or responsibility for the accuracy, completeness, or usefulness of any information, apparatus, product, or process disclosed, or represents that its use would not infringe privately owned rights. Reference herein to any specific commercial product, process, or service by trade name, trademark, manufacturer, or otherwise does not necessarily constitute or imply its endorsement, recommendation, or favoring by the United States government or Lawrence Livermore National Security, LLC. The views and opinions of authors expressed herein do not necessarily state or reflect those of the United States government or Lawrence Livermore National Security, LLC, and shall not be used for advertising or product endorsement purposes.

This work performed under the auspices of the U.S. Department of Energy by Lawrence Livermore National Laboratory under Contract DE-AC52-07NA27344. 


\title{
Improved capture $\gamma$-ray libraries for nuclear applications
}

\author{
A. M. Hurst ${ }^{1}$, B. W. Sleaford ${ }^{2}$, R. B. Firestone ${ }^{1}$, N. C. Summers ${ }^{2}$, Zs. Revay ${ }^{3}$, L. Szentmiklósi ${ }^{3}$, \\ S. Basunia ${ }^{1}$, T. Belgya ${ }^{3}$, J. E. Escher ${ }^{2}$, M. Krticka ${ }^{4}$ \\ ${ }^{1}$ Lawrence Berkeley National Laboratory, Berkeley, CA 94720, USA \\ ${ }^{2}$ Lawrence Livermore National Laboratory, Livermore, CA 94550, USA \\ ${ }^{3}$ Centre for Energy Research, Hungarian Academy of Sciences, Budapest, Hungary \\ ${ }^{4}$ Charles University in Prague, Prague, Czech Republic
}

\begin{abstract}
The neutron-capture reaction is of fundamental use in identifying and analyzing the $\gamma$-ray spectrum from an unknown object as it gives unambiguous information on exactly what isotopes are absorbing the neutrons. There are many applications where this can be used passively (nonproliferation), or actively where an external neutron source is used to probe an unknown assembly (planetary studies). There are known capture- $\gamma$ data gaps in the ENDF libraries used by transport codes for various nuclear applications. A new database, EGAF, containing thermal neutron-capture $\gamma$-ray data is used to improve the capture- $\gamma$ information in the ENDF libraries. For many nuclei the unresolved quasi-continuum part of the $\gamma$ cascade is not available experimentally. In this work, we have modeled this contribution using the Monte Carlo statisticaldecay code DICEBOX, in addition to improving level-scheme evaluations. For capture of higher-energy neutrons there is little experimental data available, making evaluation of modeling codes problematic. We plan to continue the DICEBOX approach through the resolved resonance region where spin and parity information is partially known. In the unresolved resonance region, and up to $20-\mathrm{MeV}$ incident neutron energy, we are applying Hauser-Feshbach models to predict the capture- $\gamma$ spectrum.
\end{abstract}

\section{Introduction}

Improved prompt capture- $\gamma$ spectra are needed for a variety of non-proliferation programs, e.g. Monte Carlo Neutron Transport Codes (MCNP) for National Security applications. Prompt $\gamma$ rays are emitted following neutron capture and can, thus, be used to unambiguously identify compositions of unknown assemblies since the observed capture $\gamma$ rays are themselves unique signatures of the elements/isotopes contained within the absorbing medium. Until recently, however, no reliable prompt neutron-capture $\gamma$-ray database existed for this type of analysis [1]. Since capture- $\gamma$ data are an essential component for many basic and applied scientific purposes, a series of thermal-capture measurements were undertaken at the Budapest Reactor on all elemental targets corresponding to $Z=1-83,90$, and 92, with the exception of helium and promethium. This work led to the development of the Evaluated Gamma-ray Activation File (EGAF) and has been evaluated and published as part of an International Atomic Energy Agency (IAEA) coordinated research project [2].

For low- $Z$ isotopes, neutron-decay schemes are likely near complete, however, for medium-heavy mass isotopes there may remain considerable gaps in the complete knowledge of the decay scheme. Furthermore, the contribution of feeding to the observed low-lying states from the quasi continuum is difficult to resolve experimentally for many nuclei. To account for this feeding and supplement the experimental data available in the EGAF database, the Monte Carlo statistical-decay code DICEBOX [3] has been utilized. New independent measurements of the total radiative thermal neutron-capture cross section $\left(\sigma_{0}\right)$ can then be extracted as the sum of experimentally measured partial-capture $\gamma$-ray cross sections feeding the ground state directly, in addition to the DICEBOX-modeled contribution from the 
continuum that feeds the ground state. Also, the comparison between DICEBOX simulations and the experimentally-determined cross sections in the EGAF database allows for optimization of the capturestate spin distribution as well as improved evaluations of the nuclear structure information that can ultimately be communicated back through the Evaluated Nuclear Structure Data File (ENSDF) [4] and the Reference Input Parameter Library (RIPL) [5], a nuclear reaction database containing reformatted structure information from ENSDF.

A future long-range goal of this work is to develop comprehensive neutron-data libraries with complete capture- $\gamma$ information covering incident-neutron energies from the thermal region, through to the resolved and then unresolved resonance regions, all the way up to $20 \mathrm{MeV}$, the Hauser-Feshbach regime. This information is vital for neutron transport calculations and will be disseminated through the Evaluated Nuclear Data File (ENDF) [6]. Unfortunately, very little experimental data are available for prompt $\gamma$-ray emission resulting from higher-energy neutron capture. To address this problem, future neutron-time-of-flight measurements are being planned. The DICEBOX approach may then also be adopted in the resolved-resonance region where spin and parity information of the $l \geq 1$ resonances is partially known, while Hauser-Feshbach models [7] will be used to treat the remaining part of the high-energy neutron-capture $\gamma$-ray spectrum.

In these proceedings we are presenting results from a series thermal-capture measurements using enriched tungsten targets. This new information will be communicated through EGAF, ENSDF, and RIPL, and will form part of revised, more accurate capture- $\gamma$ library that will be made available through the ENDF database for nuclear applications.

\section{Experimental Setup}

Partial neutron-capture $\gamma$-ray cross sections were measured with the mirror-guided thermal-neutron beam at the 10-MW Budapest Research Reactor [8,9]. A thermal flux of approximately $7.8 \times 10^{7} \mathrm{n} \cdot \mathrm{cm}^{-2} \cdot \mathrm{s}-1$ was incident upon samples of isotopically-enriched tungsten oxide compounds. An elemental sample of tungsten oxide was also irradiated with a thermal flux of $2 \times 10^{6} \mathrm{n} \cdot \mathrm{cm}^{-2} \cdot \mathrm{s}-1$. The samples were prepared in powder form and held in the beam line in Teflon bags. Due to the proximity of the evacuated target-sample holder, a distance some $35 \mathrm{~m}$ away from the reactor wall, a low-background environment permits for the detection of primary and secondary capture- $\gamma$ rays. The Prompt Gamma Activation Analysis (PGAA) setup [10,11] is located at this target station and, in its simplest operational mode, comprises a single Compton-suppressed $n$-type high-purity germanium (HPGe) detector with a closedend coaxial-type geometry. The HPGe detector is positioned approximately $23.5 \mathrm{~cm}$ from the targetsample holder. The energy calibration and counting efficiency of the HPGe detector was accomplished using a variety of standard radioactive sources spanning an energy range of $\sim 50 \mathrm{keV}$ up to $\sim 11 \mathrm{MeV}$. The $\gamma$-ray spectroscopy software package HYPERMET-PC [12] aided the generation of non-linear energycalibration and efficiency fits to the data, in addition to the peak-fitting analysis of the capture $\gamma$-ray spectrum.

Partial $\gamma$-ray cross sections were extracted from the measured peak areas for all tungsten capture$\gamma$ lines using an internal-standardization procedure which normalizes the observed intensities to wellknown comparator lines [13]. Hydrogen was used as the comparator in this case from a standard $(\mathrm{n}, \gamma)$ measurement with tungstic acid $\left(\mathrm{H}_{2} \mathrm{WO}_{4}\right)$ [14]. Cross sections for all tungsten $\gamma$ lines were then derived based on this standardization (n, $\gamma$ ) measurement. To ensure consistency over the observational range, a statistically-consistent set of normalization factors were required in five different regions encompassing the low-energy, turning-point, medium- and high-energy regions of the capture-(n, $\gamma)$ spectrum. Furthermore, this requirement of consistency between low-, medium, and high-energy normalization factors for each sample, allowed for determination of independent sample thicknesses by considering, and correcting peak intensities for, $\gamma$-ray self absorption within the tungsten oxide powders themselves. The attenuation coefficients were calculated using data from XMUDAT [15] which is based on the prescription outlined in Ref. [16]. 


\section{Statistical Model Calculations}

The thermal neutron-capture $\gamma$ cascade has been simulated by calculating theoretical feedings to the experimentally observed low-lying levels using the Monte Carlo code DICEBOX [3]. This program is based on the generalization of the extreme statistical model, proposed by Bohr [17], in the formation and decay of the compound nucleus. Thermal neutron capture is an $s$-wave process $(l=0)$ whereupon the compound product is formed with an excitation energy corresponding to its neutron separation energy. Using this information, and within this theoretical framework, the DICEBOX calculation is then constrained according to an experimental decay scheme up to a defined energy, referred to as the critical energy $E_{\text {crit }}$. All experimental information characterizing the decay scheme including level energies, spins and parities, $\gamma$-ray transition energies and their corresponding branching ratios as well as internalconversion coefficients, are regarded as complete and accurate up to $E_{\text {crit }}$. The code then considers the region between $E_{\text {crit }}$ and the neutron separation energy as the quasi continuum and uses a random discretization of an a priori known level density (LD) formula $\rho\left(E, J^{\pi}\right)$ and photon strength function (PSF) $f^{(X L)}\left(E_{\gamma}\right)$ to generate $\gamma$-ray transitions from within this region to the low-lying levels in the experimental decay scheme. A partial radiation width $\Gamma_{i f}^{(X L)}$ describing the transition probability for a given $\gamma$-ray decay with an energy $E_{\gamma}=E_{i}-E_{f}$ is then assumed to be a random choice from a Porter-Thomas distribution [18], centred on a mean value given by

$$
\left\langle\Gamma_{i f}^{(X L)}\right\rangle=\frac{f^{(X L)}\left(E_{\gamma}\right) \cdot E_{\gamma}^{2 L+1}}{\rho\left(E_{i}, J_{i}^{\pi_{i}}\right)} .
$$

In equation (1), $\rho\left(E_{i}, J_{i}^{\pi_{i}}\right)$ represents the level density near the initial level $i$ and $X L$ denotes the multipolarity of the transition involved; selection rules are fully accounted for in the generation of the partial widths. The system of partial radiation widths fully describing the decay properties of the low-lying levels as well as the randomly-generated levels of the quasi continuum is known as a nuclear realization. Decay properties of the levels involved differ according to each independent nuclear realization i.e. levelscheme simulation, and thus, generate statistical fluctuations in the simulated level feedings. By using an appropriate number of realizations DICEBOX can be used to determine the nature of the Porter-Thomas fluctuations involved, and therefore, provide an estimate of the uncertainty attributed to the statistical nature of the decay processes. These proceedings describe calculations involving 50 separate nuclear realizations, with each realization comprising 100,000 capture-state $\gamma$-ray decay cascades.

Phenomenological models have been used to describe the influence of the PSF and LD on the simulated level feedings. Since the dominant decay mode from the capture state at the neutron separation energy is via an electric dipole transition $(E 1)$, Lorentzian-based models are used to describe the shape of the corresponding giant dipole electric resonance (GDER) observed in photonuclear reactions. Several different GDER-based PSFs are implemented in the DICEBOX code [3] and all were tested in this analysis. The Enhanced Generalized Lorentzian (EGLO) model $[19,20]$, which is dependent on both $\gamma$-ray energy as well as nuclear temperature, was found to reproduce the experimental absorption data for tungsten rather well and was employed as the principal E1 PSF in this work. The GDER parametrizations for the tungsten isotopes were taken from RIPL [5]. However, other models were also tested, and even the simpler Brink-Axel (Standard Lorentzian) [21,22] model-dependent upon $\gamma$-ray energy alone-was found to produce statistically invariant results cf. the EGLO model. The next most important capture-state decay mode is through an $M 1$ magnetic dipole transition, although this mode is significantly hindered by approximately an order of magnitude compared to the $E 1$ contribution. For the $M 1$ PSF, Lorentzian-based models were considered and tested, however, due to the paucity of experimental data available for the giant dipole magnetic resonance (GDMR), in a somewhat general sense and certainly in the case of tungsten, a single-particle (SP) PSF model was adopted. A final consideration was also given to the PSF for the much weaker $E 2$ electric quadrupole primary transitions. In this case, a global parametrization $[23,24]$ has been used to describe the expected shape of the giant quadrupole electric resonance (GQER) for the adopted PSF model. The simulations in this work made use of both 
the constant temperature formula (CTF) and back-shifted Fermi gas (BSFG) models for the adopted level density. The parametrizations for these models were taken from Ref. [25]. All possible combinations of PSF and LD were tested in these calculations.

\section{Results}

The four major tungsten isotopes i.e. ${ }^{182} \mathrm{~W}(26.50 \%),{ }^{183} \mathrm{~W}(14.31 \%),{ }^{184} \mathrm{~W}(30.64 \%)$, and ${ }^{186} \mathrm{~W}$ $(28.43 \%)$, have been investigated through a series of isotopically-enriched tungsten $\left({ }^{182,183,186} \mathrm{~W}\right)$ and elemental tungsten $\left({ }^{184} \mathrm{~W}\right)$ thermal neutron-capture measurements. The extracted cross sections have then been compared to theoretical predictions using the DICEBOX code to simulate the thermal-capture $\gamma$-ray cascade. The quality of the results are assessed in terms of how well the modeled predictions for level populations compare with the experimental depopulation data for a given level. Thus, plotting these two quantities against each other gives an immediate impression of the validity of the statistical model in addition to the quality and completeness of the experimental data. This approach can then be used to postulate on the existence of missing $\gamma$ rays, and indeed, search for them in the collected $(\mathrm{n}, \gamma)$ spectra. The statistical model can also be used to test various spin-parity $\left(J^{\pi}\right)$ assignments for levels which have tentative assignments and even suggest new values in the case of strongly populated levels with no known $J^{\pi}$ assignments. Other structural information may also be extracted such as confirmation of mixing ratios for $\gamma$-ray transitions with mixed multipolarities.

A complete summary of this analysis of the major tungsten isotopes, including new independent measurements of the total radiative thermal neutron-capture cross sections and $\gamma$-emission probabilities, will be made available in Ref. [26], while earlier developments in this work may be found in Refs. [2729]. In these proceedings, we would like to highlight some of the methods that we have adopted in improving the capture- $\gamma$ spectrum and structural information. Results from ${ }^{186} \mathrm{~W}(\mathrm{n}, \gamma)$ will be used as a case-in-point illustration. For the ${ }^{187} \mathrm{~W}$ compound a cut-off energy corresponding to $E_{\text {crit }}=900 \mathrm{keV}$ was determined. Beneath this value of $E_{\text {crit }}, 40$ low-lying levels (including the ground state) are known to exist. Figure 1 shows the corresponding population-depopulation plot for this nucleus up to $E_{\text {crit }}$. In Fig. 1(a) it can be seen that population and depopulation data are all in good agreement with the exception of the low-lying level at $E_{\mathrm{ex}}=364.2 \mathrm{keV}$. This level is reported in ENSDF (and therefore, RIPL) to have only two $\gamma$ rays depopulating it at around 162.7 and $286.9 \mathrm{keV}[4,5]$. However, the plot clearly indicates that the DICEBOX-simulated population for the $364.2-\mathrm{keV}$ level is significantly greater than experimental depopulation corresponding to the extracted partial $\gamma$-ray cross sections for the 162.7- and 286.9-keV transitions. Since the experimental data for all other levels compare well for this nucleus, the implication is that the statistical model provides an accurate simulation for the ${ }^{187} \mathrm{~W}$ capture$\gamma$ decay scheme, and it is more likely that there could be something wrong or incomplete with regards to the experimental data for the $364.2-\mathrm{keV}$ level. The $J^{\pi}=9 / 2^{-}$assignment for this level is firmly established $[4,5]$ and so an alternative scenario could be there is a missing $\gamma$-ray transition deexciting this level that would otherwise provide the extra amount of intensity needed to reach agreement with the DICEBOX calculation. Fig. 1(b) shows that inclusion of a $\sim 14-\mathrm{keV}$ transition to the 350.43-keV level improves agreement between simulation and experiment dramatically; all data now fall along the slope indicating good agreement between population and depopulation for levels up to $E_{\text {crit }}$. The cross section for the $\sim 14-\mathrm{keV}$ transition has been estimated based on expected population predicted by DICEBOX. In fact, the recent work of Bonadarenko et al. [30] suggests that such a transition should exist due to inferred coincidence relationships, despite not actually observing the transition directly. The course of this work has, indeed, highlighted several other instances where low-energy transitions, that have not been observed in nuclear structure experiments, may well exist according to statistical model expectations [26]. It is important that such transitions should be considered in establishing an optimized and more-complete capture- $\gamma$ library. 
(a)

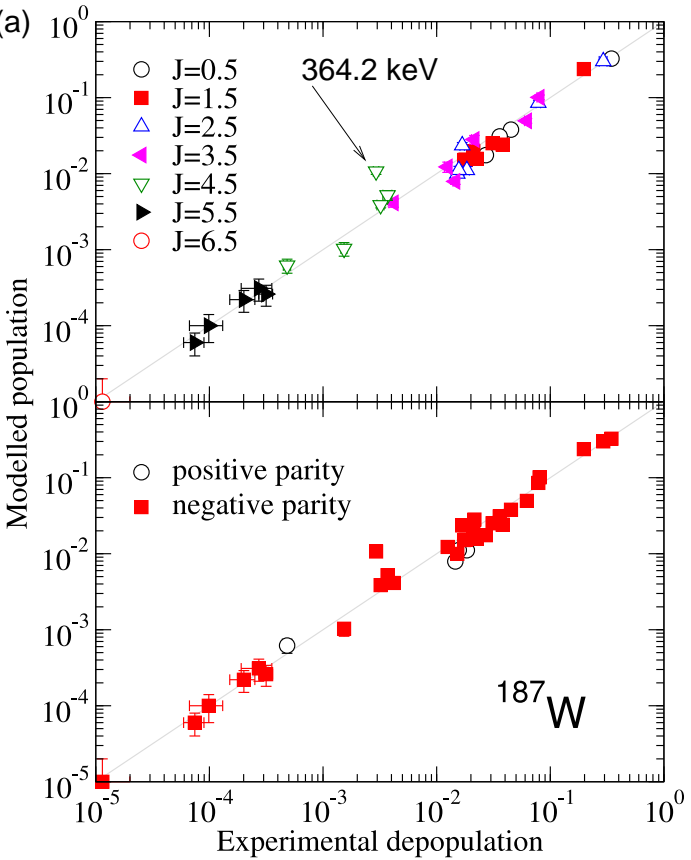

(b)

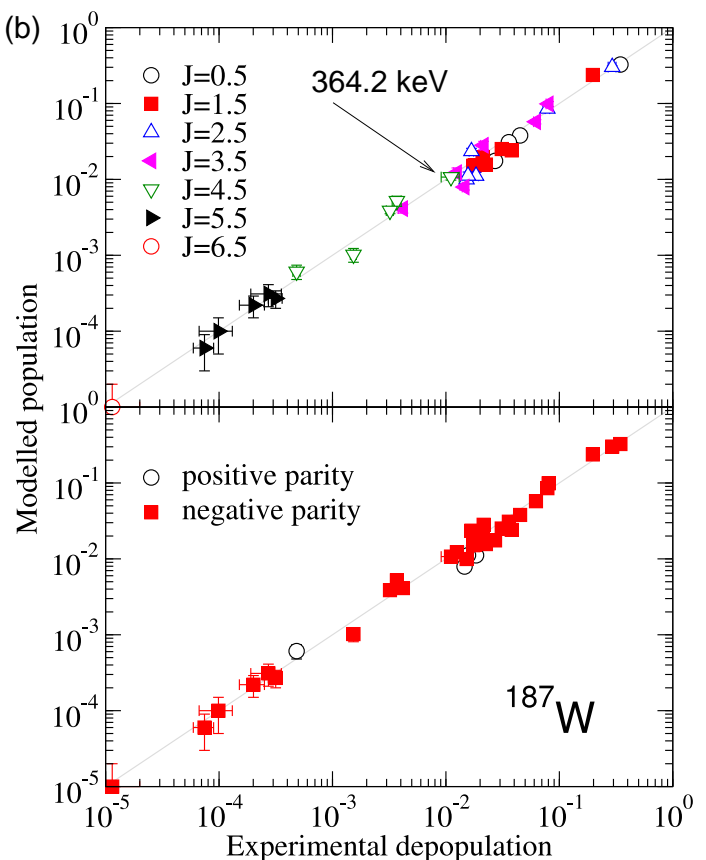

Fig. 1: Simulated populations (DICEBOX) plotted against experimental depopulations (capture- $\gamma$ cross sections) for low-lying levels in ${ }^{187} \mathrm{~W}$ up to $E_{\text {crit }}=900.0 \mathrm{keV}$ : (a) poor agreement between simulation and experiment for the 364.2-keV level; (b) good agreement between simulation and experiment for all levels.

\section{Conclusion}

Partial $\gamma$-ray cross sections have been measured for the major tungsten isotopes from a series of thermal neutron-capture experiments performed at the Budapest Research Reactor using isotopically-enriched and elemental tungsten samples. The quality and completeness of these data have been investigated by comparison to theoretical predictions of thermal capture onto tungsten isotopes using the Monte Carlo statistical-decay code DICEBOX. The combined experimental and theoretical effort has led to an increase in the cut-off energy $E_{\text {crit }}$ compared to what is currently reported in RIPL [5] for all four tungsten compounds $\left({ }^{183,184,185,187} \mathrm{~W}\right)$ investigated in this work. Furthermore, a more complete database of thermal-capture $\gamma$-rays and energy levels has been deduced for each of the compounds in this study, along with improved nuclear structure information such as confirmation of tentative $J^{\pi}$ assignments in addition to newly-proposed assignments for certain levels. This information is currently in preparation for a forthcoming publication [26] and will be communicated back into the EGAF [2], ENSDF [4], and RIPL [5] libraries, and eventually disseminated as part of a complete capture- $\gamma$ library to be made available through the ENDF [6] neutron-data library.

In the future we would like to extend our measurements with tungsten into the resolved-resonance and higher-energy neutron-capture regions, up to $20 \mathrm{MeV}$. These measurements will be accompanied by further statistical-model calculations using DICEBOX (resolved-resonance region) and Hauser-Feshbach (high-energy capture) techniques with the ultimate goal of generating a complete capture- $\gamma$ library to be published through ENDF. This, in turn, will provide a more robust and reliable input-data-set for many applications using MCNP.

\section{Acknowledgments}

This work was performed under the auspices of the University of California, supported by the Director, Office of Science, Office of Basic Energy Sciences, of the U. S. Department of Energy at Lawrence Berkeley National Laboratory under Contract DE-AC02-05CH11231, and by the U. S. Department of Energy by Lawrence Livermore National Laboratory under Contract DE-AC52-07NA27344, the Hungar- 
ian project OMFB 0018/2006 NAP VENEUS05, and by the research plan MSM 0021620859 supplied by the Ministry of Education of the Czech Republic. The operations staff at the Budapest research reactor are gratefully acknowledged.

\section{References}

[1] B. W. Sleaford et al., J. Kor. Phys. Soc. 59, 1473 (2011).

[2] R. B. Firestone et al., IAEA STI/PUB/1263, 251 (2007).

[3] F. Becvár, Nucl. Instrum. Methods Phys. Res. A 417, 434 (1998).

[4] Evaluated Nuclear Structure Data File, National Nuclear Data Center, Brookhaven National Laboratory. http://www. nndc.bnl.gov/ensdf/.

[5] R. Capote et al., Nucl. Data Sheets, 110, 3107 (2009). http://www-nds.iaea.org/RIPL-3/.

[6] Evaluated Nuclear Data File, an electronic database of evaluated nuclear data maintained by the IAEA. http://www-nds.iaea.org/exfor/endf.htm/.

[7] Walter Hauser and Herman Feshbach, Phys. Rev. 87, 366 (1952).

[8] L. Rosta et al., Physica B, 234-236, 1196 (1997).

[9] L. Rosta, Appl. Phys. A 74, S52-S54 (2002).

[10] Zs. Révay et al., Nucl. Instrum. Methods Phys. Res. B 213, 385 (2004).

[11] L. Szentmiklósi et al., J. Radioanal. Nucl. Chem. 286, 501 (2010).

[12] Hypermet-PC Version 5.01 (v512), Institute of Isotopes (1995-97), Budapest, Hungary. http://www.iki.kfki.hu/nuclear/hypc/.

[13] Zs. Révay and G. L. Molnár, Radiochim. Acta 91, 361 (2003).

[14] L. Szentmiklósi, private communication.

[15] XMuDat: Photon attenuation data on PC, Version 1.0.1 of August 1998. http://www-nds.iaea.org/publications/iaea-nds/iaea-nds-0195.htm/.

[16] J. H. Hubbel and S. M. Seltzer, Tables of X-Ray Mass Attenuation Coefficients and Mass EnergyAbsorption Coefficients $1 \mathrm{keV}$ to $20 \mathrm{MeV}$ for Elements $Z=1$ to 92 and 48 Additional Substances of Dosimetric Interest, NISTIR 5632 (1995). http://physics.nist.gov/PhysRefData/XrayMassCoef/cover.html/.

[17] N. Bohr, Nature (London), 137, 344 (1936).

[18] C. E. Porter and R. G. Thomas, Phys. Rev. 104, 483 (1956).

[19] J. Kopecky and M. Uhl, Phys. Rev. C 41, 1941 (1990).

[20] J. Kopecky, M. Uhl, and R. E. Chrien, Phys. Rev. C 47, 312 (1993).

[21] D. M. Brink, Ph. D. Thesis, University of Oxford (1955).

[22] P. Axel, Phys. Rev. 126, 671 (1962).

[23] J. Speth and A. van der Woude, Rep. Prog. Phys. 44, 719 (1981).

[24] W. V. Prestwitch, M. A. Islam, and T. J. Kennett, Z. Phys. A 315, 103 (1984).

[25] T. von Egidy and D. Bucurescu, Phys. Rev. C 72, 044311 (2005).

[26] A. M. Hurst et al., to be submitted to Phys. Rev. C (2012).

[27] A. M. Hurst et al., J. Korean Phys. Soc. 59, 1491s (2011).

[28] A. M. Hurst et al., AIP Conf. Proc. 1342, 24 (2011).

[29] A. M. Hurst et al., Eur. Phys. J. Web of Conferences 21, 10005 (2012).

[30] V. Bondarenko et al., Nucl. Phys. A811, 28 (2008). 